\title{
Systems thinking and ethics in public health: a necessary and mutually beneficial partnership
}

\author{
Diego S. Silva ${ }^{1} \cdot$ Maxwell J. Smith ${ }^{2,3} \cdot$ Cameron D. Norman ${ }^{3,4}$
}

Published online: 13 June 2018

(c) Monash University 2018

\begin{abstract}
Systems thinking has emerged as a means of conceptualizing and addressing complex public health problems, thereby challenging more commonplace understanding of problems and corresponding solutions as straightforward explanations of cause and effect. Systems thinking tries to address the complexity of problems through qualitative and quantitative modeling based on a variety of systems theories, each with their own assumptions and, more importantly, implicit and unexamined values. To date, however, there has been little engagement between systems scientists and those working in bioethics and public health ethics. The goal of this paper is to begin to consider what it might mean to combine systems thinking with public health ethics to solve public health challenges. We argue that there is a role for ethics in systems thinking in public health as a means of elucidating implicit assumptions and facilitating ethics debate and dialogue with key stakeholders.
\end{abstract}

Keywords Public health ethics $\cdot$ Social networks $\cdot$ Systems dynamimcs $\cdot$ Systems thinking

Consider the following two fictitious cases:

Case one The Public Health Agency of Canada (PHAC) is in the midst of updating guidelines for the management of multidrug resistant tuberculosis (MDR-TB) and the authors vehemently disagree about how long a person with MDR-TB should remain in isolation after they have been diagnosed and started treatment. From the

Diego S. Silva

dsilva@sfu.ca

1 Faculty of Health Science, Simon Fraser University, Blusson Hall, Room 11300, 8888

University Drive, Burnaby, BC V5A 1S6, Canada

2 School of Health Studies, Faculty of Health Sciences, Western University, London, Ontario, Canada

3 Dalla Lana School of Public Health, University of Toronto, Toronto, Canada

4 CENSE Research + Design, Toronto, Canada 
literature, the authors know that isolation of those with MDR-TB after diagnosis can range anywhere from 2 weeks to 6 months (Lange et al. 2014). One author says that, given the severity of MDR-TB, the public should be placed at little to no risk of infection. Another argues that, although protecting the public from infection is important, so too is the free movement of persons with MDR-TB and that they should only have to remain in isolation for as short a duration of time as possible despite a slightly higher risk of harm to the public.

Case two A public health unit in southern California struggles to design programs and strategies to promote healthy eating in a local low-income Latino community (Kaufman and Karpati 2007). Given their interpretation of the academic literature, coupled with their own experiences working with this community, the public health workers believe that in order to implement a successful program, they will have to contend with the following: first, that family bonds-which are particularly important to many Latinos in Southern California-are maintained and strengthened through the sharing of foods; and two, that food insecurity in this economically poorer community means that families will procure lower-quality foods (e.g., fatty or sugary foods) at their local corner stores on credit from the owners of the corner stores (who are also local Latinos) when they can no longer afford fresh fruits and vegetables from supermarkets.

$* * * * *$

Most of those who work in public health or in associated fields, like public health ethics, will readily concede that, much like the two cases outlined above, attending to the public's health is complex and consists of attempting to address a wide variety of interdependent challenges that variably lead to ill health at a population level. Moreover, many of these same individuals will readily admit that there are normative, often ethical, questions and assumptions to clarify when trying to solve these complex public health problems. Much less frequently, though, do those working in public health and public health ethics (especially the latter) stop to consider what it means for something to be complex, and as importantly, what it means to take into account complexity when trying to discern the morally right, or best, or defensible solution to a public health problem.

In the past decade or so, systems thinking, i.e., the field of research and practice related to the study of systems, has been more readily used in public health to help address the complex problems that belie simple solutions. And yet, there has been little to no discussion in public health ethics about how systems thinking might aid in our work, or how public health ethics can work together with systems scientists to better describe and build models that are sensitive to the ethical underpinnings of social systems.

The goal of this paper is therefore to consider what it might mean to integrate systems thinking and public health ethics to address complex public health challenges. In their efforts to improve public health, we argue that public health ethics scholars must strive to understand both how public health problems are embedded within complex social systems and how the activities of governments across sectors work to promote or undermine 'solutions' to such problems; doing so requires acknowledging the complexity of such systems but also working with systems scientists to develop better models of systems for programmatic planning and policy change. 
In this paper we argue that there is a role for ethics in systems thinking in public health and that this role is twofold: first, those practicing and conducting research in public health ethics ought to be aware of systems thinking due to the emerging use of various systems theories and their associated methods in public health research and practice. Systems theories may provide an opportunity to help public health ethicists think through complex and potentially counterintuitive challenges in public health. Second, we argue that there is an opportunity to explicitly situate ethics as a pillar of systems thinking in public health by working with systems scientists and other stakeholders in defining the boundaries of a given system and adding ethical nuance to the various components of a given theory or model. If one of the ongoing struggles for public health ethics (and bioethics, more generally) is making ethics more relevant to policy-makers and practitioners, working alongside those who develop models of public health systems may provide a concrete avenue for meaningful participation. This paper is divided into three sections. First, we provide an overview of the basic terms and ideas used in systems thinking. Second, we introduce the emergence of systems thinking in public health. Third, we describe the relationship between systems thinking in public health and public health ethics and argue that there exists an explicit role for public health ethics in systems thinking and vice versa, while returning to the two cases outlined above as examples of this relationship.

Two points of clarification are necessary before beginning: first, one of the main tools that systems researchers use are models (i.e., systems researchers will often create descriptive and predictive models of systems based upon certain assumptions and beliefs about the world). While our discussion certainly touches upon issues related to models and modeling, our focus is on systems thinking and not modeling per se; the act of modeling may have an overlapping, but unique, set of normative issues that are important to consider but are ultimately beyond the scope of this paper. Second, while engagement between public health ethics and systems thinking in public health would in our view be mutually beneficial, we fully acknowledge that systems thinking is not a panacea for public health problems, or that systems thinking will always provide clarity for those working through ethical issues in public health. Rather, we argue that there exists the potential for mutually beneficial learning and scholarly engagement between systems theorists and those working in public health ethics, which could work to augment the traditional methods used in public health ethics, such as philosophical argumentation and empirical research.

\section{Systems thinking and complexity}

Complex social systems bring together many factors, which, by quirks of history, have been compartmentalized into isolated intellectual fields. The barriers between disciplines must melt away if we are successfully to cope with complex systems. Within the same system we must admit the interactions of the psychological, the economic, the technical, the cultural, and the political. The interactions among these are often more important than the internal content of any one alone. 
- Jay W. Forrester (1969: 109)

The complexity inherent in public health problems, and the complexity involved in trying to solve these problems, may seem self-evident. Attempts to actually tame, understand, and control the complex nature of public health, however, requires introducing new ways of thinking. Systems thinking and the science underlying it have been identified as a means of conceptualizing and addressing complex public health challenges (Leischow et al. 2008; Mabry et al. 2010). A complex problem is one that has many overlapping and interacting sources of influence and variation that manifests in different ways across contexts, at different time scales, and is not amenable to simple, linear solutions (i.e., easily identifiable cause and effect relationships, like that of a cue and a billiard ball) (Williams and Hof 2016). Classifying and conceptualizing problems in public health as 'complex' has intuitive appeal given the invariable interconnectedness between public health and other sectors of public policy and practice, in addition to the field's multifaceted focus on the social determinants of health, the prevention of diseases, and health promotion. A system is “... a whole the functioning of which depends on its parts and the interactions between those parts" (Jackson 2003: 3). Systems thinking refers to the field of research and practice related to the study of systems (also referred to as systems science), which includes theories, methods, and approaches aimed at describing, changing, and developing systems.

Public or social problems, which would include those in public health, can be divided into three broad categories that exist along a continuum: simple, complicated, and complex (Snowden 2005). Simple problems are those where the concept of 'best practice' can be applied, where there is a known pathway between cause and effect, and guides akin to recipes can be developed based on evidence. Complicated problems arise when certain causal structures are difficult to predict, or where mapping the various interactions and parts of a system itself requires time and expertise (Plsek 2003), and where 'good practice' is more appropriate. Good practice might mean using 'good enough' knowledge based on approximate evidence and experience to inform action, but with reduced certainty compared to 'best practice' where there is a clearly defined solution based on strong evidence. In this case, there may be multiple pathways to solving a complicated problem, but the problem most often can be diagnosed and addressed with some confidence that the solution will be satisfactory.

Finally, complex systems are those where the interactions between the parts cannot be reduced to the function or intended role of the parts themselves, and where even understanding the parts and interactions may not secure future understanding or success, which ultimately requires adopting a holistic approach. The various theories about complex systems include common descriptive similarities. First, complex denotes a system with many interacting parts where the rules of interaction are potentially unknown and are dynamic. Second, systems are dynamic whereby they are constantly changing, adapting, and evolving relative to various internal and external environmental stimuli. Third, causality in complex systems functions in a non-linear manner (i.e., the cause and effect are rarely in proportion), there may exist time delays between causes and effects, and the causes and effects might 
occupy vastly different regions of a system. Fourth, complex systems are often characterized by feedback mechanisms or 'loops', where outputs or changes in some interactions or in some parts of the system will alter the manner in which the system processes said change and reacts or functions in the future.

Although there are several diverse and prominent systems theories, one key point of convergence is the acknowledgement that any attempt to solve complex problems requires solutions that take seriously the interconnected and sometimes counterintuitive relationships between the various stakeholders, resources, and values of a given system. How values shape systems is perhaps not fully understood, but the role of normative ethics is beginning to be considered a key to systems thinking: as Sterman notes, "Systems thinking requires us to examine issues from multiple perspectives, to expand the boundaries of our mental models, to consider the longterm consequences of our actions, including their environmental, cultural, and moral implications" (Sterman 2006).

As mentioned earlier, systems science seeks to understand systems through the development of models, which includes the examination of mental models and the generation of visual representations based often on theory, qualitative and quantitative simulations, or blended forms of data. Choosing what is or is not part of a model (i.e., a model's boundaries) is an analytic step that usually follows from the particular theory itself. For example, a theory like soft-systems methodology relies more heavily on qualitative mental maps or models than theories like system dynamics, which tends to use quantitative data to best plot the boundaries of a system and the interaction of the various parts. Much like ethics, thinking about systems is an iterative process. In other words, there is no such thing as a perfect model that perfectly describes the world; rather, models are used as heuristics that are constantly changing and evolving relative to new information and new ideas by the systems scientists themselves. But the role of modeling should not be underestimated; the ability to model complex systems is meant to help researchers and decision-makers begin to understand the complexity inherent in some systems and to expose the oftentimes counterintuitive interactions within systems.

\section{Systems thinking and public health}

\subsection{The various systems of public health}

In recent years there has been increased interest from researchers, practitioners, and policy-makers in public health to explicitly use systems thinking to help solve, or begin to address, complex problems within public health. As Trochim and colleagues write: "More [public health] scholars are studying and writing on the topic, more research is emphasizing a systems view, and ambitious attempts are under way to focus practitioners on improving overall system performance" (2006: 540). Many public health problems are considered to be complex; for example, issues related to arresting the spread of infectious diseases or curbing smoking rates requires an understanding of human behaviour, the social determinants of health, etiology of diseases, statistics, political structures, and laws, to name only a few relevant 
considerations. Establishing boundaries and the interactions within and between the various parts of a public health problem contained in these systems is itself a complex task (Ulrich and Reynolds 2010), as potential solutions may have counterintuitive and unforeseen consequences both for population health and for the other social aims pursued by other sectors (e.g., reductions in overall smoking rates may not be equally distributed among persons of different socioeconomic status; barring tobacco sales in corner stores may have economic and labour force implications). As Midgley notes: “. . the whole concept of public health is founded on the insight that health and illness have causes or conditions that go beyond the biology and behavior of the individual human being" (2006: 466).

Public health's broad focus and mandate requires engagement from a diverse workforce who must learn to work across boundaries created by discipline, sector, geography, method, and practice. Moreover, those who are affected by public health measures include a wide and varied group of individuals with their own viewpoints and beliefs that, one might argue, ought to be accounted for in deliberations (e.g., marginalized populations versus those in positions of power, various races and ethnicities, etc.). Leischow and Milstein write that one of the appeals of systems thinking for public health is the fact that public health requires, and systems thinking may provide, an opportunity for, “... transdisciplinary thinking as well as active engagement of those who have a stake in the outcome to govern the course of change" (2006: 403). Thinking and working across disciplines is not a new idea in public health (or bioethics and public health ethics), nor is the belief that it is important to engage and encourage participation from the various stakeholders who are affected by a given problem and its potential solution. The idea is to work across disciplines and engage with various stakeholders to build better models of the complex problems based on systems theories in order to begin to try to solve, in an iterative manner, the many challenges in public health.

\subsection{Conceptualizing the public health problem and the system}

Boundaries can be created by mental models of the system under investigation. The manner in which public health problems are conceived through mental models shapes solutions; change the mental model and you change the possible solutions that emerge. For example, assuming one identifies challenges associated with meeting optimal vaccination rates for seasonal influenza, how a person describes said challenges would differ based on a variety of factors, including one's disciplinary background and occupation. For instance, a virologist or immunologist may conceptualize the problem of vaccine uptake as microbiological in nature and will strive to make a vaccine as effective as possible while minimizing potential side-effects. A public health nurse on the other hand might conceptualize the problem as predominantly clinical in nature (i.e., how to engage a patient, educate him or her if any knowledge gaps exist, or even address biases the patient might have, all relative to the nurse's knowledge and beliefs as a health professional). A behavioural scientist in a public health unit who is charged with devising the most effective means of vaccine uptake among and within various populations might conceptualize the problem differently still, given knowledge about 
structural supports for, and barriers to, vaccination that may stem from different laws and policies that govern his or her public health unit. Someone from operations management might see the problem as that of supply chain management. Others who do not support vaccines in general or believe that a vaccine might not be useful for seasonal influenza might not see vaccines uptake as the problem at all.

That identifying and conceptualizing a problem differs based on multiple contextual factors (e.g., culture, disciplinary background, economic factors, etc.) is, or seems like, a truism; the further point, however, is that how different individuals try to solve problems in public health are often (a) based on potentially false mental models that make use of some simple notion of linear causality or only one particular vantage point; or (b) that even if an individual acknowledges that a problem defies simple solutions, he or she is unable to understand how to go about solving problems that they willfully acknowledge are complex. First, one might argue that, when conceptualizing a public health challenge, people tend to use models of behaviour that view the cause and effect of behaviour change as linear (i.e., that clear and known inputs produce clear and known outputs). For example, traditional health behaviour theories (e.g., transtheoretical model, health belief model, etc.) identify a set of inputs within a particular process or set of processes that, when altered, can promote positive or negative changes in health behaviour; however, despite some evidence as to their effectiveness in guiding health promotion efforts, such theories of health behaviour change may be criticized as being overly simplistic as they largely disregard social factors and fail to account for the manner in which a person's environment can shape his or her health behaviour (Jackson 2003). There are many other ways in which problems may be misunderstood beyond presuming simple and linear notions of causality (e.g., temporal ambiguity with regard to causes and effects, counterintuitive effects, etc.). As Plsek and Greenhalgh argue, 'Newton's 'clockwork universe' in which big problems can be broken down into smaller ones, analyzed, and solved by rational deduction has strongly influenced both the practice of medicine and leadership... [b]ut the machine metaphor lets us down badly when no part of the equation is constant, independent, or predictable" (2001: 625). In other words, some problems may not be solvable by referring to its constitutive parts, since some problems require acknowledging the interdependence between the various parts.

The previously mentioned 'simple' models of behaviour change are used under the guise of tackling one part of the problem at a time (i.e., trying to reduce the problem to more manageable linear pieces, thereby inadvertently ignoring the interrelated aspects of public health problems). As Rittel and Webber argue in their seminal paper on 'wicked problems' in planning: “...it has become less apparent where problem centers lie, and less apparent where and how we should intervene even if we do happen to know what aims we seek [emphasis in the original]" (1973: 157).

\section{Systems thinking and public health ethics}

Despite the sporadic use of terms like 'complexity' and 'systems' in public health ethics, to date there are few explicit attempts to evaluate how systems theories can help people reason through ethical challenges in public health. What few examples 
exist in the literature have generally used the idea of 'systems' as a rhetorical device and do not engage with systems theories themselves (e.g., social network theory, system dynamics, soft-systems methodology, etc.). There is also little acknowledgement of a connection between systems thinking and public health ethics in the academic and grey literature. Among the few examples where ethics is discussed in this literature is in the use of critical systems heuristics (Ulrich and Reynolds 2010, and in that case the discussion is largely focused on the role of decisions in framing boundaries (i.e., boundary critique) in systems decisions and analyses). In this section we argue that systems thinking can assist those working in public health ethics conceptualize details of particular public health challenges and, moreover, that public health ethics and bioethics could potentially help systems scientists build models of health systems that more explicitly take into account values.

\subsection{What systems thinking can do for public health ethics}

The main concern of public health ethics is to attend to the ethical issues related to the health of populations, and in particular marginalized or vulnerable groups, within the context of their environments, including the cultural, economic, social, and political realities that shape people's lives. For example, Powers and Faden's non-ideal account of justice consists of, in part, redressing reinforcing inequalities that affect a person's or group's well-being, arguing that "[i]nequalities beget inequalities and existing inequalities... can compound, sustain, and reproduce a multitude of depravations in well-being..." (2006: 72). Goldberg furthers Powers and Faden's theory by arguing that the focus on the individual's actions in health promotion activities in the USA, for example, ignores the apparent fact that "... the prevalence of one social disadvantage makes it significantly more likely that members of that population will experience other social disadvantages" (2012: 108). The need to look at the context in which health inequalities occur also suggests that greater attention must be paid to the relationships between people and the influence they may have on each other. Thus, those working in public health ethics generally acknowledge that tackling health inequalities, and public health challenges more broadly, in an ethical manner means, in part, being aware of the interconnected nature of individuals and their relationships with their environments, which would seem to lend itself to being open to incorporating systems thinking as part of the analysis needed to achieve their aims.

To date, much of the bioethics and public health ethics literature has used systems thinking primarily as a metaphor to help researchers and practitioners think through ethical problems in medicine and public health; they do not actually engage with particular systems theories. For example, McRae and colleagues (2008) argue that systems thinking can be used to guide "boardroom to bedside" (314) thinking and to bridge "the artificial gap between organizational and clinical domains" (314); they suggest that systems ideas, such as having a "clear organizational mandate" (316) and staying "engaged with the "real world" (316), will help bioethicists solve ethical conundrums. Roberts and colleagues call for a "systems bioethics" that takes into account the various "complexities of the biological, medical, societal, and 
ethical questions in some of the same ways as the emerging field of systems biology" by acknowledging that "there is no such thing as a stand-alone bioethical issue or stand-alone bioethical analysis" (2006: 28). Hyder and colleagues have recently written about "health systems research" which is about "translating efficacious interventions into effective practice at the population level" and thereby "blurs the distinction between research and nonresearch processes", but do not necessarily adopt the ideas behind systems thinking per se (2014: 36).

Using systems thinking as a metaphor to guide thinking in bioethics and public health ethics is an important first step in acknowledging the complexity of health for both individuals and populations, especially marginalized populations who are most burdened by health inequalities. However, addressing ethical challenges in public health and affecting change that will help redress mutually reinforcing inequalities might require moving beyond using systems thinking as mere metaphor. In other words, it will require actually understanding the science that describes relationships between individuals, groups, and their environments. Some scholars working in public health ethics have begun to acknowledge the potential benefits for ethics researchers and practitioners to learn from various systems theories. For example, Battle-Fisher's recently published book begins to investigate the social and ethical implications of adopting systems thinking in health policy debates (Battle-Fisher 2015). While this contribution is welcomed, more work remains to be done in terms of robustly engaging with systems thinking in public health ethics. Wilson, in turn, has argued that

...unless we can factor in a sufficient understanding of the inner wiring of the complex systems we are trying to reform, our attempts as philosophers to suggest how institutions should be reformed will either be dismissed as unworkable by those who are responsible for delivering services on the ground - or worse! - they may be implemented, and cause the unintended consequences and alienation of professionals... (2009: 187).

Wilson provides two important insights: first, assuming that the prescriptions made by those working in public health ethics are adopted as policy or practice, the instituted changes could be ignored outright by public health practitioners if the complexity of the problem that affects those working on the frontlines in health promotion and public health policy are not fully appreciated. Second, assuming public health ethics recommendations are adopted and actually implemented in policy and practice, merely applying traditional ethics thinking to nonlinear problems (even while acknowledging that the problems in public health are nonlinear and complex) could have unintended consequences that may worsen or create new problems (Wilson 2014). For instance, applying the harm principle (commonly appealed to as a foundational value of public health-Upshur 2002) to justify smoking bans in mental health centers might disproportionately harm persons with schizophrenia who derive some neurological and social benefits to smoking, a nuance often overlooked in both the academic literature and health policy (Silva 2011; Silva et al. 2013).

Systems thinking can provide the models for mapping and understanding the environments in which policies are considered, potentially reducing the harms of consequences from those actions by modeling what possible and probable 
outcomes can be connected to a particular activity. Public health ethics can use the models of systems thinking to better understand the complexity of the public health problems they hope to address through ethical arguments and policy and practice recommendations. In reality, using empirical evidence to inform ethics deliberations is not new to bioethics or public health ethics; it is now commonplace and one is expected to ground one's arguments in empirical evidence. Thus, systems thinking offers a new source of evidence and information to help guide our normative deliberations and arguments. The novelty would be to use systems modeling as a means of testing one's conclusions in order to anticipate the possible downstream effects of certain policy or practice prescriptions. If we in public health ethics want to inform policy and practice, as noted by Wilson above, ethics scholars should likely want to use all tools to better understand the potential repercussions of ethics prescriptions, which can be used in conjunction with traditional methods of analysis within bioethics. Using the models produced by the various systems theories is meant to complement, and not replace, the traditional forms of analysis in bioethics and public health ethics.

Let us now return to Case One, that of the feuding authors of PHAC's MDRTB guidelines: their disagreement concerned whether it was more important to subject individuals with MDR-TB to long isolation periods after diagnosis and initiation of treatment in order to protect the public, or shorter isolation periods in order to promote the freedom of movement of those with MDR-TB. We happily concede that systems thinking does not help resolve the debate regarding what is of greater moral importance, public safety or personal liberties; however, systems modeling might be able to present what the ramifications of each moral stance may be. Imagine that the feuding authors have access to a modeler at PHAC and that they use system dynamics theory (i.e., a theory about how varied and multiple complex systems function in non-linear fashion) to model the transmission of TB infection, which in previous studies described how various strains of drug resistant TB in a community with variable latent periods affects the rate of infectivity (Castillo-Chavez and Feng 1997; Castillo-Chavez and Song 2004), or how primary healthcare physicians' access or lack of access to specialized medical expertise in Pakistan affects the care of TB patients (Malik et al. 2014). The guideline authors could, in theory, build with their modeler colleague variables that would test the rate of infectivity given greater or lesser duration of isolation of persons with MDR-TB in different parts of Canada in conjunction with other scientific, social, and economic variables. Again, this would not settle their ethics dispute, but would afford them the opportunity to discuss the potential consequences of their viewpoints in an informed manner, rather than merely imagining what those consequences might be, which in turn would give the authors new information to consider and perhaps modify their perspectives on isolation. To return to one of Wilson's points articulated above, it would provide a real way of testing whether there are any unintended consequences that are morally unpalatable to either party. 


\subsection{What public health ethics can do for systems thinking}

If systems thinking has hereto been ignored in public health ethics, ethics too has only been considered by systems scholars in passing and never seriously as one of the pillars of systems thinking. The fundamental questions that guide systems thinking are, at least partially, normative, and specifically, questions of ethics. Public health ethics, therefore, is in the ideal position to help address these questions.

Writing within the context of system dynamics, Pruyt and Kwakkel argue that the four questions that systems theorists ask themselves (or ought to ask themselves) prior to research are: "Who matters? What matters? What time horizon matters? What are the boundaries of the system/model to be considered?" (2007: 4). They also acknowledge that these questions are fundamentally ethical in their scope. Defining the relevant parties ('who matters') and the scope of dispute or debate ('what matters') serve as part of the foundation to any ethical analysis, as are determining what normative considerations count or ought to count ('what time horizons and boundaries matter') within a given debate. These four questions presuppose that there is a clearly articulated problem, which itself contains ethical considerations, as noted earlier. In other words, the very act of articulating and describing a challenge in public health is itself a normative endeavour, which would prove vital to any systems theorist trying to help address a public health problem.

A clear example of how public health ethics scholars can contribute to systems thinking can be found in Mulvaney-Day and Womack's treatment of research on obesity (2009). According to the authors, some prominent and important research on obesity reduction strategies are based on social network theory (i.e., in which behaviour is understood in light of the relationships between individuals and across a number of relationships of various strengths of influence). Using existing research on social network theory and obesity, Mulvaney-Day and Womack argue that traditional behaviour change programs that try to curb obesity are too narrow and individualistic, and fail to account for the role of relationships in promoting healthy eating. They argue that the idea of relational autonomy (i.e., where personal values and personal identity are chosen or shaped in the context of relationships) provides a normative ethics grounding for social network research into obesity. They argue that, despite the potential negative effects that social circles can have in promoting obesity-inducing behaviour, these same social networks can be exploited to promote healthy eating, e.g., by using peer support programs.

Returning to our second case discussed at the outset of this paper, and extending Mulvaney-Day and Womack's relational autonomy approach, the idea that family bonds are strengthened through the sharing of food, which at times might lead to overeating, perhaps can be used by California public health officials as a point of education, where several types of behaviour change interventions could be attempted. The owners of local corner stores, where many Latinos get their food, can be engaged to try to leverage their status in their communities to improve access to healthy food. Thus, the values of mutual care and concern, values that ought to be promoted by those who subscribe to relational accounts of autonomy, can be used to support more obesity strategies on the basis of complexity and community, and away from individualistic methods of behaviour 
change. Mulvaney-Day and Womack's work provides an example of how ethics and systems thinking can work together to try to improve the health of populations and persons. The ethical idea of relational autonomy can give social network researchers an important normative concept when trying to implement changes through various public health levers; in turn, social network theory provides relational notions of autonomy with an empirical grounding and greater real-world nuance.

Public health ethicists are trained to ask questions and provide answers to questions about who and what should matter in a particular public health debate, and what factors ought to be considered when evaluating what course of action should be undertaken. As a field, we are well-situated to work alongside systems theorists to help build models that consider the implicit ethical questions that underlie systems thinking. In particular, given the increased popularity of empirical bioethics, and specifically the history of both arguing for and conducting stakeholder or public engagement activities to help inform and resolve bioethics challenges (e.g., pandemic influenza planning - Silva et al. 2012; Smith et al. 2012; Smith and Silva 2015), those public health ethicicsts already engaging in empirical bioethics research might be in the ideal position to help solicit and understand the perspectives of various stakeholders in order to help inform systems modeling and the value-laden prescriptions made in the context of systems thinking more generally.

\section{Conclusion}

Bioethics prides itself on its interdisciplinary nature. Systems thinking constitutes a newer disciplinary perspective that bioethics ought to bring into the fold. In public health, systems thinking has the potential to bring together established disciplines like epidemiology, biostatistics, law, policy, and health promotion so as to help reduce entrenched health inequalities and address negative social determinants of health. In addition to the important role that ethics must play in public health itself, those working in public health ethics have a unique opportunity to collaborate with systems scientists in a mutually beneficial manner that can enrich public health policies and programs. If an ethics for public health approaches complex population health challenges as a set of simpler causal parts to each be considered and addressed in turn, then it will fail to take seriously the complexity of such challenges and the emerging systems approaches to addressing them. Systems thinking can provide those working in public health ethics with a new tool to understand complex problems, and ethics can provide systems scientists with a deeper understanding of the values that necessarily underpin systems theories and models.

Acknowledgements We would like to thank James Wilson for his important and helpful comments on an earlier version of this manuscript. We would also like to thank the Fondation Brocher; Diego Silva and Maxwell Smith were fellows there when much of the conceptualization of this paper occurred. 


\section{References}

Battle-Fisher, M. 2015. Application of Systems Thinking to Health Policy and Public Health Ethics. New York: Springer.

Castillo-Chavez, C., and Z. Feng. 1997. To treat or not to treat: the case of tuberculosis. Journal of Mathematical Biology 35: 629-656.

Castillo-Chavez, C., and B. Song. 2004. Dynamical models of tuberculosis and their applications. Mathematical Biosciences and Engineering 1 (2): 361-404.

Forrester, J.W. 1969. Urban Dynamics. Cambridge: MIT Press.

Goldberg, D.S. 2012. Social justice, health inequalities and methodological individualism in US health promotion. Public Health Ethics 5 (2): 104-115.

Hyder, A.A., A. Rattani, C. Krubiner, et al. 2014. Ethical review of health systems research in lowand middle-income countries: a conceptual exploration. American Journal of Bioethics 14 (2): 28-37.

Jackson, M.C. 2003. Systems Thinking: Creative Holism for Managers. West Sussex, UK: Wiley.

Kaufman, L., and A. Karpati. 2007. Understanding the sociocultural roots of childhood obesity: Food practices among latino families of Bushwick, Brooklyn. Social Science and Medicine 64: 2177-2188.

Lange, C., I. Abubakar, J.W. Alffenaar, et al. 2014. Management of patients with multidrug-resistant/ extensively drug-resistant tuberculosis in Europe: A TBNET consensus statement. European Respiratory Journal 44 (1): 23-63.

Leischow, S.J., and B. Milstein. 2006. Systems Thinking and Modeling for Public Health Practice. American Journal of Public Health 96 (3): 403-405.

Leischow, S.J., A. Best, W.M. Trochim, et al. 2008. Systems thinking to improve the public's health. American Journal of Preventive Medicine 35 (2 SUPPL): 196-203.

Malik, A., C. Willis, S. Hamid, et al. 2014. Advancing the application of systems thinking in health: Advice seeking behavior among primary health care physicians in Pakistan. Health Research Policy and Systems. https://doi.org/10.1186/1478-4505-12-43.

Mabry, P.L., S.E. Marcus, P.I. Clark, et al. 2010. Systems science: A revolution in public health policy research. American Journal of Public Health 100 (7): 1161-1163.

McRae, S.K., E. Fox, and A. Slowther. 2008. Clinical ethics and systems thinking. In The Cambridge Textbook of Bioethics, ed. P.A. Singer and A.M. Viens, 313-321. Cambridge, UK: Cambridge University Press.

Midgley, G. 2006. Systemic intervention for public health. American Journal of Public Health 96 (3): 466-472.

Mulvaney-Day, N., and C. Womack. 2009. Obesity, identity and community: Leveraging social networks for behavior change in public health. Public Health Ethics 2 (3): 250-260.

Plsek, P.E. (2003) Complexity and the Adoption of Innovation in Health Care. Washington, D.C.: The National Institute for Health Care Management Foundation and the National Committee for Quality Health Care. Retrieved November 6, 2017. http://www.nihcm.org/pdf/Plsek.pdf.

Plsek, P.E., and T. Greenhalgh. 2001. The challenge of complexity in health care. BMJ 323: 625-628.

Powers, M., and R. Faden. 2006. Social Justice: The Moral Foundations of Public Health and Health Policy. Oxford, UK: Oxford University Press.

Pruyt, E., and Kwakkel, J. (2007) Combining system dynamics and ethics: towards more science?" System Dynamics Society. Retrieved November 6, 2017. http://www.systemdynamics.org/conference s/2007/proceed/papers/PRUYT232.pdf.

Rittel, H.W.J., and M.M. Webber. 1973. Dilemmas in a general theory of planning. Policy Sciences 4: $155-169$.

Roberts, J.S., J. Maienschein, and M.D. Laubichler. 2006. Systems bioethics and stem cell biology. Journal of Bioethical Inquiry 3: 19.

Silva, D.S. 2011. Smoking bans and persons with schizophrenia: a straightforward use of the harm principle? Public Health Ethics 4(2): 143-148.

Silva, D.S., M.J. Smith, and R.E.G. Upshur. 2013. Disadvantaging the disadvantaged: when public health policies and practices negatively affect marginalized populations. Canadian Journal of Public Health 104(5): 410-412.

Silva, D.S., J.L. Gibson, A. Robertson, et al. 2012. Priority setting of ICU resources in an influenza pandemic: a qualitative study of the Canadian public's perspectives. BMC: Public Health 12: 241. 
Smith, M.J., and D.S. Silva. 2015. Ethics for pandemics beyond influenza: ebola, drug-resistant tuberculosis, and anticipating future ethical challenges in pandemic preparedness and response. Monash Bioethics Review 33(2-3): 130-147.

Smith, M.J., C. Bensimon, D. Perez, et al. 2012. Restrictive measures in an influenza pandemic: a qualitative study of public perspectives. Canadian Journal of Public Health 103(5): e348-e352.

Snowden, D.J. 2005. Multi-ontology sense making: a new simplicity in decision making. Informatics in Primary Care 13: 45-53.

Sterman, J.D. 2006. Learning from evidence in a complex world. American Journal of Public Health 96 (3): 505-514.

Trochim, W.M., D.A. Cabrera, and B. Milstein. 2006. Practical challenges of systems thinking and modeling in public health. American Journal of Public Health 96 (3): 538-546.

Ulrich, W., and M. Reynolds. 2010. Critical systems heuristics. In Systems Approaches to Managing Change: A Practical Guide, ed. M. Reynolds and S. Holwell, 243-292. London: Springer.

Upshur, R.E.G. 2002. Principles for the justification of public health intervention. Canadian Journal of Public Health 93 (2): 101-103.

Williams, B., and S. Hof. 2016. Wicked Solutions: A Complex Approach to Complex Problems. North Carolina: Lulu.com.

Wilson, J. 2009. Towards a normative framework for public health ethics and policy. Public Health Ethics 2 (2): 184-194.

Wilson, J. 2014. Embracing complexity: Theory, cases and the future of bioethics. Monash Bioethics Review 32: 3-21. 